\title{
Erratum to: Wave-function-based characteristics of hybrid mesons
}

\author{
Nosheen Akbar ${ }^{\mathrm{a}}$, Bilal Masud ${ }^{\mathrm{b}}$, and Saba Noor ${ }^{\mathrm{c}}$ \\ Centre For High Energy Physics, University of the Punjab, Lahore (54590), Pakistan
}

Original article: Eur. Phys. J. A (2011) 47: 124, DOI: 10.1140/epja/i2011-11124-2

Received: 31 March 2014

Published online: 31 July 2014 - (C) Società Italiana di Fisica / Springer-Verlag 2014

1) The normalized radial wave functions in figs. 1, 5 and 6 were plotted by using the normalization condition $\int R^{\star} R=1$ instead of $\int U^{\star} U=1$. Here we revised the calculations for radial wave functions by using the correct normalization condition. All other results presented in the paper remain unaffected, except for the radial wave functions at origin in the fourth column of table 10 of the original publication. The revised figures and magnitudes of radial wave functions at origin are reported in the following figures and table.

2) $\nabla^{2}$ in eq. (3) should be replaced with $\frac{d^{2}}{d r^{2}}$.

3) In eq. (4), $\left\langle r^{2}\right\rangle$ should be replaced by $\sqrt{\left\langle r^{2}\right\rangle}$.

4) In the paragraph after eq. (6), the symbol $m$ is replaced by $\mu$.

The authors apologize for these mistakes and any inconvenience caused.

Table 10. $|R(0)|^{2}$ of $c \bar{c}$ meson.

\begin{tabular}{|c|c|c|c|}
\hline Meson & $L$ & $S$ & $\begin{array}{c}\text { Our calculated normalized }|R(0)|^{2} \\
\mathrm{GeV}^{3}\end{array}$ \\
\hline$\eta$ & 0 & 0 & 1.2294 \\
\hline$J / \psi$ & 0 & 1 & 1.9767 \\
\hline$h_{c}$ & 1 & 0 & $\approx 0$ \\
\hline$\chi_{c}$ & 1 & 1 & $\approx 0$ \\
\hline
\end{tabular}

\footnotetext{
a e-mail: noshinakbar@yahoo.com

b e-mail: bilalmasud.chep@pu.edu.pk

c e-mail: sabanoor87@gmail.com
} 


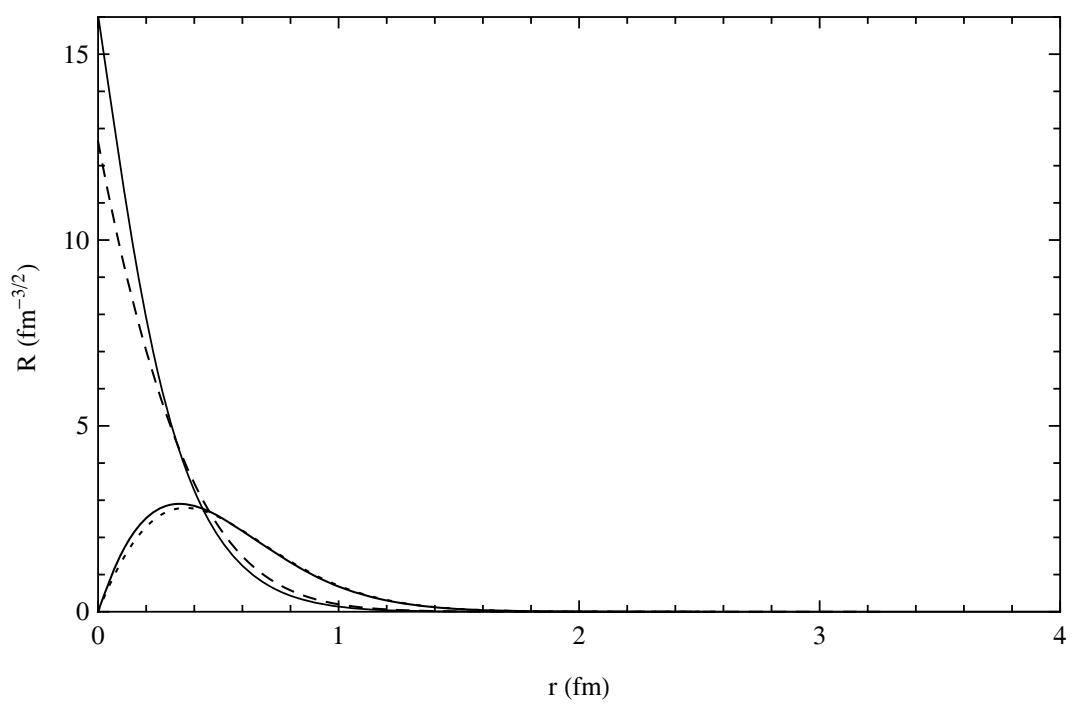

Fig. 1. Revision of fig. 1.

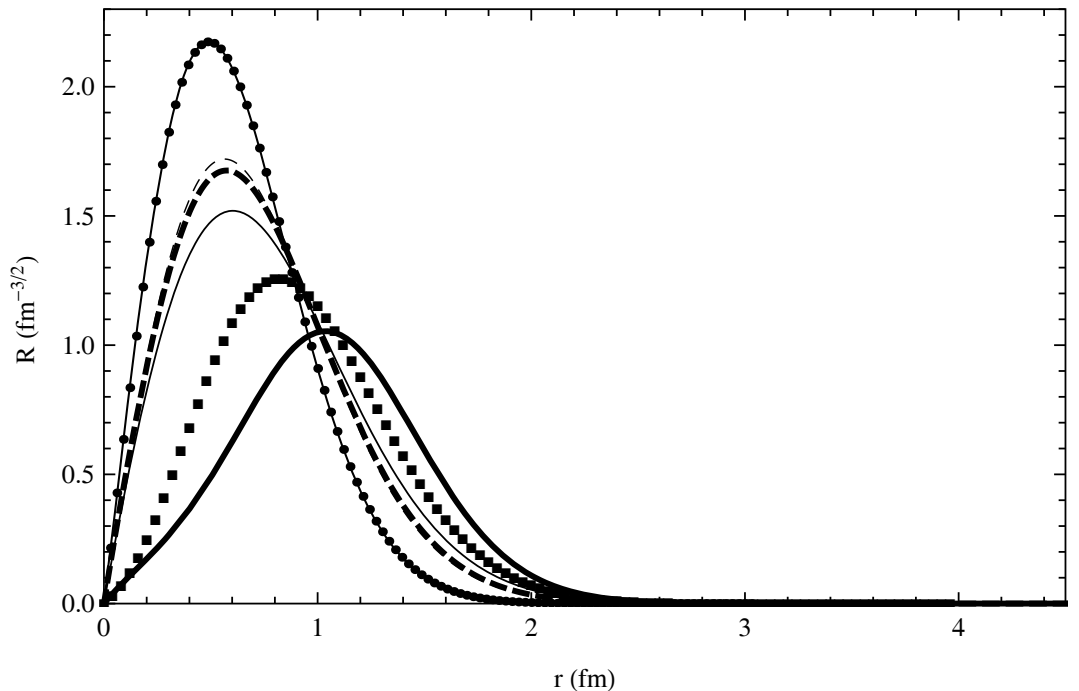

Fig. 5. Revision of fig. 5 .

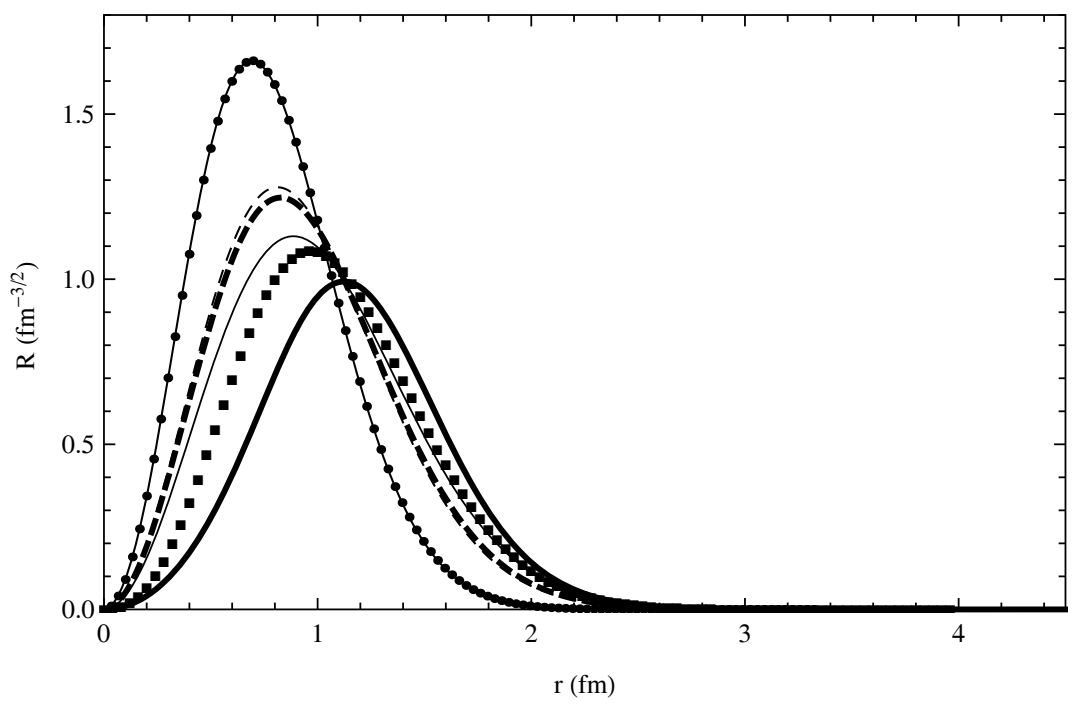

Fig. 6. Revision of fig. 6 . 